\title{
Promoción de las competencias ligadas al pensamiento computacional a través del aprendizaje basado en el desarrollo de proyectos
}

\section{Promotion of skills related to computational thinking in times of pandemic}

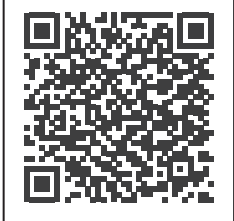

Palabras clave: competencias, computacional, pensamiento, problemas, prototipos, proyectos, solución.

Articulo de investigación:

Fecha de recepción:

$30 / 11 / 2020$

Fecha de aceptación:

$12 / 04 / 2021$

Esta publicación se encuentra bajo licencia:

Creative Commons

Reconocimiento-

NoComercial-

SinObraDerivada 4.0

Internacional

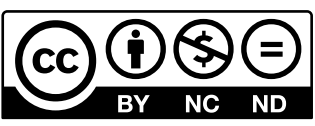

Juan Antonio Sánchez-Márquez; Mónica Móndelo-Villaseñor²; Elizabeth Mena-Avilés33; Ana Jovita Méndez-Martínez ${ }^{4}$

\section{Resumen}

Problemática: la mayor complejidad de los problemas actuales demanda que los estudiantes fortalezcan sus competencias ligadas a la solución de problemas. Si enseñamos a los estudiantes a resolver problemas, ellos adquirirán independencia y confianza para enfrentar retos futuros. Objetivos: este proyecto se enfoca en la promoción de las competencias del pensamiento computacional a través del desarrollo de proyectos. Materiales y métodos: los alumnos trabajaron en el desarrollo de proyectos en equipo, haciendo uso de roles. Durante el desarrollo de los proyectos, los alumnos debieron procesar información, modelar los prototipos iniciales, evaluar las fallas e incorporar las ideas originales basadas en sus propios procesos

1 Doctor en Ciencias en Ingeniería Química, Escuela del Nivel Medio Superior de Salamanca, Universidad de Guanajuato, México. ja.sanchez@ugto.mx, ORCID: 0000-0001-9619-8901

2 Maestría en Gestión y Desarrollo, Escuela de Nivel Medio Superior de León, Universidad de Guanajuato, México.m.mondelo@ugto.mx, ORCID: 0000-00028485-055X

3 Profesora, Instituto Tecnológico y de Estudios Superiores de Monterrey, Campus León, México. elizabeth.mena@tec.mx, ORCID: 0000-0002-8849-2390 4 Profesora, Unidad Profesional Interdisciplinaria de Ingeniería, Campus Guanajuato, Instituto Politécnico Nacional, México. ajmendez@ipn.mx, ORCID: 0000-0002-0267-3466 


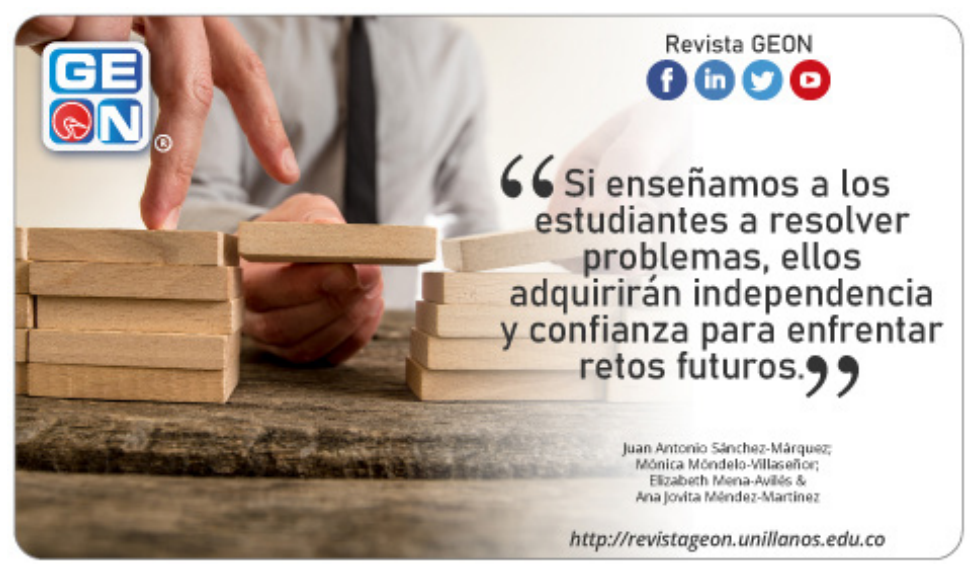

creativos. Resultados: los resultados muestran que el principal reto que identificaron los alumnos fue el de generar acuerdos al interior de los grupos que les permitieran hacer adecuaciones a los prototipos y llegar a las versiones finales de estos. Discusión: en este proyecto, los alumnos fueron expuestos a situaciones que emularon ambientes reales de trabajo, en donde ellos debían desarrollar consensos al tiempo que identificaban una ruta sistemática para resolver problemas. Conclusiones: este tipo de proyectos genera un espacio idóneo para que los alumnos asuman roles, se comuniquen eficientemente, aprendan entre ellos y desarrollen soluciones innovadoras bajo un ambiente real de aprendizaje colaborativo. Contribución/ originalidad: este proyecto permitirá que los docentes cuenten con una metodología de solución de problemas que podrán compartir con sus estudiantes a fin de que estos puedan aplicarla a la solución de problemas emulando situaciones que los alumnos encontrarán durante su desarrollo profesional y personal.

Palabras clave: competencias, computacional, Cómo citar este artículo:
Toreference this article:

Sánchez Márquez, J. A. Móndelo Villaseñor, M., Mena Avilés, E., \& Méndez Martínez, A. J. . (2021). Promoción de las competencias ligadas al pensamiento computacional a través del aprendizaje basado en el desarrollo de proyectos. Revista GEON (Gestión, Organizaciones Y Negocios), 8(1), e-294. https://doi. org/10.22579/23463910.294 pensamiento, problemas, prototipos, proyectos, solución.

Códigos JEL: 121 Analysis of Education

\section{Abstract}

Problematic: The greater complexity of current problems demands that students strengthen their 
Cómo citar este artículo /

Toreference this article:

Sánchez Márquez, J. A.,

Móndelo Villaseñor, M., Mena Avilés, E., \& Méndez Martínez,

A. J. . (2021). Promoción de

las competencias ligadas al

pensamiento computacional a

través del aprendizaje basado en

el desarrollo de proyectos. Revista

GEON (Gestión, Organizaciones Y

Negocios), 8(1), e-294. https://doi. org/10.22579/23463910.294 skills related to problem solving. If we teach students to solve problems, they will gain independence and confidence to face future challenges. Goals: This project focuses on promoting computational thinking skills through project development. Materials and methods: The students worked on the development of team projects, using roles. During their development, the students had to process information, model the initial prototypes, evaluate flaws, and incorporate original ideas based on their own creative processes. Results: The results show that the main challenge identified by the students was to generate agreements within the groups that would allow them to adjust the prototypes and reach their final versions. Discussion: In this project, the students were exposed to situations that emulated real work environments, where they had to develop consensus while identifying a systematic route to solve problems. Conclusions: This type of project creates an ideal space for students to assume roles, communicate efficiently, learn from each other, and develop innovative solutions in a real collaborative learning environment. Contribution/ originality: This project will allow teachers to have a problem-solving methodology that they can share with their students so that they can apply it to solving problems by emulating situations that students will encounter during their professional and personal development.

Keywords: Computational; Problems; Projects; Prototypes; Skills; Solution; Thinking.

\section{Introducción}

Una de las competencias más importantes para la vida es, sin duda, la resolución de problemas. Desde edades muy tempranas, los seres humanos nos enfrentamos a diversos desafíos que ponen a prueba nuestro carácter y nuestros conocimientos y habilidades. Debido a lo anterior, resulta evidente que es indispensable que aprendamos a resolver problemas desde pequeños y que fortalezcamos y estimulemos nuestras habilidades a lo largo de toda nuestra formación académica. En el contexto actual, bajo esquemas de educación a distancia, debemos fomentar el desarrollo de competencias ligadas a la solución de problemas. Si enseñamos a 
los estudiantes a resolver problemas por sí mismos, ellos adquirirán independencia y confianza para enfrentar problemas futuros. De este modo, cuando los jóvenes se enfrenten a un problema que no puedan resolver, en lugar de frustrarse buscarán la manera idónea para solucionarlo, poniendo a prueba su imaginación, sus habilidades y sus conocimientos.

Al final del día, esto deberá traducirse en confianza en sí mismo y en un caminar más auténtico a lo largo de su propia trayectoria académica. No debemos perder de vista que la resolución de problemas es el primer camino para el aprendizaje, la planificación y la socialización, ya que al enfrentar los problemas buscamos apoyo para solucionarlos y en esta búsqueda se presentan oportunidades ricas de interacción, autorrealización y crecimiento personal y profesional (Rivera Cerpa, et al, 2016).

Antes de la contingencia sanitaria que nos ha tocado vivir en los últimos meses, los retos de la docencia estaban encaminados a la incorporación gradual de la tecnología en los procesos educativos y a la integración de las herramientas tecnológicas y didácticas en las diferentes áreas de estudio. La extensión de la pandemia alrededor del planeta hizo evidente, en los países en vías de desarrollo, que se carecía de la infraestructura necesaria para garantizar la continuidad del proceso de enseñanza-aprendizaje y el cumplimiento de los objetivos planteados en la planeación en materia de educación (Morales Espíndola, et al, 2020). . Incluso, en algunos casos, como el de nuestro país, se carecía en los diferentes niveles educativos de un plan de contingencia que permitiera, a alumnos y maestros, continuar con los aprendizajes planteados en sus materias.

A pesar de todos los pormenores expuestos, es preciso mencionar que la contingencia sanitaria nos ha permitido vivir una situación única de aprendizaje en materia de educación. Por un lado, la labor del docente se ha dignificado al hacerse evidente el papel de los maestros en el proceso de enseñanza-aprendizaje y el rol que ellos desempeñan para evitar que la educación se deshumanice. También ha sido notorio el nivel de compromiso de los docentes que, a pesar de la falta de recursos e infraestructura, han buscado los medios para apoyar a los alumnos en su proceso de aprendizaje.

Al inicio de la emergencia sanitaria, la principal preocupación de los docentes se centró en la adecuación de los contenidos a la nueva modalidad educativa, a fin de que los alumnos pudieran acceder a ellos y a las experiencias de aprendizaje propuestas. Durante esta primera etapa, todo fue vertiginoso, improvisado $y$, hasta cierto punto, caótico. Por un lado, los docentes con mayor experiencia en la integración de herramientas tecnológicas y pedagógicas pudieron transitar de manera muy "suave" a la nueva modalidad, que, cabe decir, no era propiamente educación a distancia, sino más bien una adaptación súbita del esquema presencial al virtual.

Ya para el segundo semestre del año pasado, los docentes contaron con 
una mayor experiencia y capacitación en el uso de recursos y herramientas tecnológicas. Es en este punto en donde la preocupación de los docentes no se enfocó más en la presentación y disposición de los contenidos o en la conexión con sus estudiantes (salvo los casos que implican condiciones socioeconómicas desfavorables). Para este momento, las preguntas clave giraban en torno a garantizar que lo que enseña se aprende y en qué grado el conocimiento es integrado por el alumno. De igual modo, en este punto se ha puesto especial atención en reconocer el valor de la interacción presente en los procesos educativos presenciales, interacción que en la virtualidad se puede ver comprometida. Una prueba de esto último son las nuevas funciones presentes en diversas plataformas asociadas al uso de microsalas para el trabajo en pequeños grupos virtuales.

Aun cuando muchos de estos aspectos han representado verdaderos retos para los docentes, es justo decir que la contingencia sanitaria ha hecho posible la incorporación súbita de herramientas tecnológicas al proceso educativo y ha reforzado la idea de que las herramientas tecnológicas son un catalizador poderoso para el cambio, actualización y mejoramiento de los procesos educativos (López, 2008). Es preciso mencionar en este punto que para poder evaluar el impacto real de la incorporación efectiva de las tecnologías en el proceso de enseñanza-aprendizaje se ha propuesto la integración de modelos de integración que sean útiles tanto para para clasificar las actividades donde se emplean tecnologías como para entender a fondo el impacto de estas (García-Utrera et al., 2014).

De igual modo, la incorporación súbita de la tecnología a los procesos educativos ha impulsado el reconocimiento de la importancia de incorporar el pensamiento computacional en el proceso de enseñanza-aprendizaje. Lo anterior se ve respaldado por estudios recientes, que proponen que las habilidades del pensamiento computacional están ligadas directamente a variables tales como los estilos de pensamiento, el éxito académico en clase y la actitud positiva frente a las clases de matemáticas (Durak \& Saritepeci, 2018). Los problemas que enfrentarán las nuevas generaciones serán cada vez más complejos y demandarán competencias ligadas a la solución de diversas problemáticas mediante el uso del pensamiento crítico y de los sistemas computacionales.

La contingencia nos ha tomado a todos por sorpresa. Nadie, en ninguna parte, estaba preparado para lo que la emergencia sanitaria planteó; sin embargo, hoy podemos decir que esta experiencia nos ha enseñado a docentes y a alumnos que la capacidad de superar circunstancias adversas es clave en la vida de una persona y que en los procesos educativos debemos enseñar a los alumnos a reconocer y valorar el papel que les toca desempeñar en su familia, su escuela y su comunidad. Es importante inculcar en los estudiantes el sentimiento de que juntos, en comunidad, se puede salir adelante. La crisis de 
la COVID-19 nos ha hecho replantear el valor de las relaciones humanas en todos los ámbitos sociales; por ende, también nos ha permitido reconocer la importancia de las relaciones humanas en la educación. No debemos permitir que los procesos educativos se deshumanicen ni hoy ni en el futuro. Debemos tomar ventaja de esta experiencia a fin de que nuestros estudiantes sean capaces de reconocer la importancia y el impacto del pensamiento crítico, lógico y computacional como herramienta clave para la solución de los problemas.

\section{Contexto teórico}

El término denominado pensamiento computacional es un concepto que se ha venido acuñando desde hace ya varias décadas. Una de las definiciones más completas y aceptadas del pensamiento computacional es la propuesta por la investigadora Jeannette Wing (2006), quien define al pensamiento computacional como: "[...] los procesos de pensamiento involucrados en la formulación de problemas y representación de sus soluciones, de manera que dichas soluciones puedan ser ejecutadas efectivamente por un agente de procesamiento de información (humano, computadora o combinaciones de humanos y computadoras)".

Hoy en día, la mayoría de los docentes definen el pensamiento computacional como la habilidad que tiene una persona para resolver problemas mediante el uso de los lenguajes de programación y de los sistemas computacionales. En esencia, el pensa- miento computacional reconoce la necesidad que se tiene actualmente de alcanzar mejores estándares de alfabetización tecnológica en los estudiantes de todos los niveles educativos a fin de reposicionar esta competencia en los ambientes de enseñanza y aprendizaje.

Si logramos fortalecer el pensamiento computacional en los procesos educativos, lograremos a su vez producir cambios positivos en la manera cómo los alumnos abordan la solución de los problemas; estaremos preparando, entonces, a nuestros estudiantes para los entornos del futuro, en donde los problemas de mayor complejidad se abordarán mediante un enfoque computacional que requiera del procesamiento de información por parte de un agente humano, computacional o híbrido. Los principios de las ciencias computacionales desafían a los estudiantes a explorar cómo la computación y la tecnología pueden impactar el mundo (García, 2012).

En este escenario, el pensamiento computacional será una competencia universal que deberá desarrollarse en las personas del mismo modo que hoy en día se hace con las matemáticas o con los idiomas. Es importante reconocer que, a medida que avanzamos en el desarrollo tecnológico, nos encontramos con problemas que son cada vez más complejos (calentamiento global, crisis económicas, crisis sanitarias, entre otros) y, por tanto, la solución de estos problemas requerirá del uso de ordenadores y del trabajo multidisciplinario y colaborativo de varios especialistas en 
diferentes áreas del conocimiento. Las proezas tecnológicas de las que hemos sido testigos en las últimas décadas han sido creadas por personas creativas que desarrollan habilidades superiores del pensamiento como el razonamiento abstracto, el pensamiento crítico, la resolución de problemas y la toma de decisiones (Zapotecatl, 2018).

El impulso del pensamiento computacional desde los niveles educativos básicos es un requisito fundamental en la formación de los individuos de la llamada sociedad de la información y el conocimiento, que permitirá hacer frente de mejor manera a los desafíos del presente siglo (Caballero \& García-Valcárcel, 2017). En estas circunstancias, resulta esencial promover los fundamentos del pensamiento computacional en los procesos educativos a fin de lograr el desarrollo sistemático de las habilidades ligadas a la descomposición de los problemas en pequeñas partes, a la detección y evaluación de fallas, el reconocimiento de patrones, a la simplificación de los elementos de un problema, al desarrollo de algoritmos (soluciones paso a paso) y a la solución de problemas con base en ideas novedosas y creativas.

El fortalecimiento de estas competencias puede ser promovido a partir del desarrollo de proyectos en equipos que representen retos reales para los estudiantes y que, sobre todo, les permitan plantear soluciones innovadoras basadas en los fundamentos del pensamiento computacional (Liu et al., 2017). Estos proyectos deben ser contextualizados en ciertas situaciones o casos que sean de relevancia e interés para los alumnos. Si cumplimos estas premisas, lograremos incrementar la motivación por la tarea encomendada y obtendremos mejores resultados en la búsqueda de soluciones.

Con base en lo expuesto, los proyectos deben tomar de base retos o desafíos de interés para los estudiantes y deben proporcionar un marco de aprendizaje que refleje el lugar de trabajo moderno (Santos et al., 2015). Además, los docentes debemos trabajar intensamente para asegurar que la atención se enfoque sobre los objetivos de aprendizaje, al tiempo que establecemos condiciones que aseguren que todos los equipos de trabajo tienen igualdad de oportunidades de cumplir con la tarea encomendada (Durán Velásquez, 2018). Es importante que durante el proceso de construcción del proyecto los docentes postulemos preguntas clave a los estudiantes, que motiven la conexión de la teoría y la práctica y que les permitan evaluar sus acciones y hacer juicios de valor. Finalmente, los docentes debemos pedir a los estudiantes que, en la medida de lo posible, validen sus argumentos y conclusiones a fin de fomentar el pensamiento crítico (Barkley, 2010).

En resumen, este proyecto se enfoca en la promoción de las competencias ligadas al pensamiento computacional a través de la metodología del aprendizaje basado en el desarrollo de proyectos. Bajo este enfoque, los alumnos deberán desarrollar proyec- 
tos en forma colaborativa que permitan el planteamiento de soluciones innovadoras al tiempo que desarroIlan habilidades socioemocionales y competencias de planificación y comunicación efectivas bajo esquemas de educación a distancia impuestos por la presente contingencia sanitaria.

\section{Materiales y métodos}

En el presente proyecto se planteó la integración de la metodología del aprendizaje basado en el desarrollo de proyectos y de los principios del pensamiento computacional. Para lograr esta integración, se atendieron los siguientes elementos metodológicos:

- Población: los grupos de trabajo estuvieron constituidos por alumnos del programa de bachillerato bivalente con especialidad en mecatrónica de la Escuela del Nivel Medio Superior de Salamanca. Los alumnos se dividieron equipos de trabajo a lo largo de las sesiones a fin de plantear soluciones a desafíos relativos a problemas específicos bajo los principios del pensamiento computacional.

- Materiales: rúbricas de evaluación, hojas de registro de información y formatos de avance del trabajo.
- Técnicas: el desarrollo de los proyectos y el planteamiento de soluciones se llevó a cabo bajo los principios del pensamiento computacional y mediante el uso de herramientas digitales, que permitieron potenciar la interacción estudiante-estudiante, maestro-estudiante y estudiante-contenido. Las soluciones a los casos de estudio y las principales impresiones de los alumnos a las estrategias implementadas fueron recabadas a través de cuestionarios digitales.

- Procedimientos: Ias fases del proyecto se detallan en la figura 1.

Toda vez que se seleccionaron los proyectos y se integraron las estrategias implementadas se procedió a configurar los equipos con base en los principios del trabajo colaborativo (figuras 3 a 5).

En este proyecto se evaluó el desarrollo de las competencias ligadas al pensamiento computacional a través de la metodología de aprendizaje basado en el desarrollo de proyectos. El impacto de dichas estrategias se evaluó a través del seguimiento de los indicadores de apropiación de las competencias ligadas al computacional (figura 6). 
Figura 1. Proceso de implementación de estrategias. Fuente: elaboración propia de los autores.

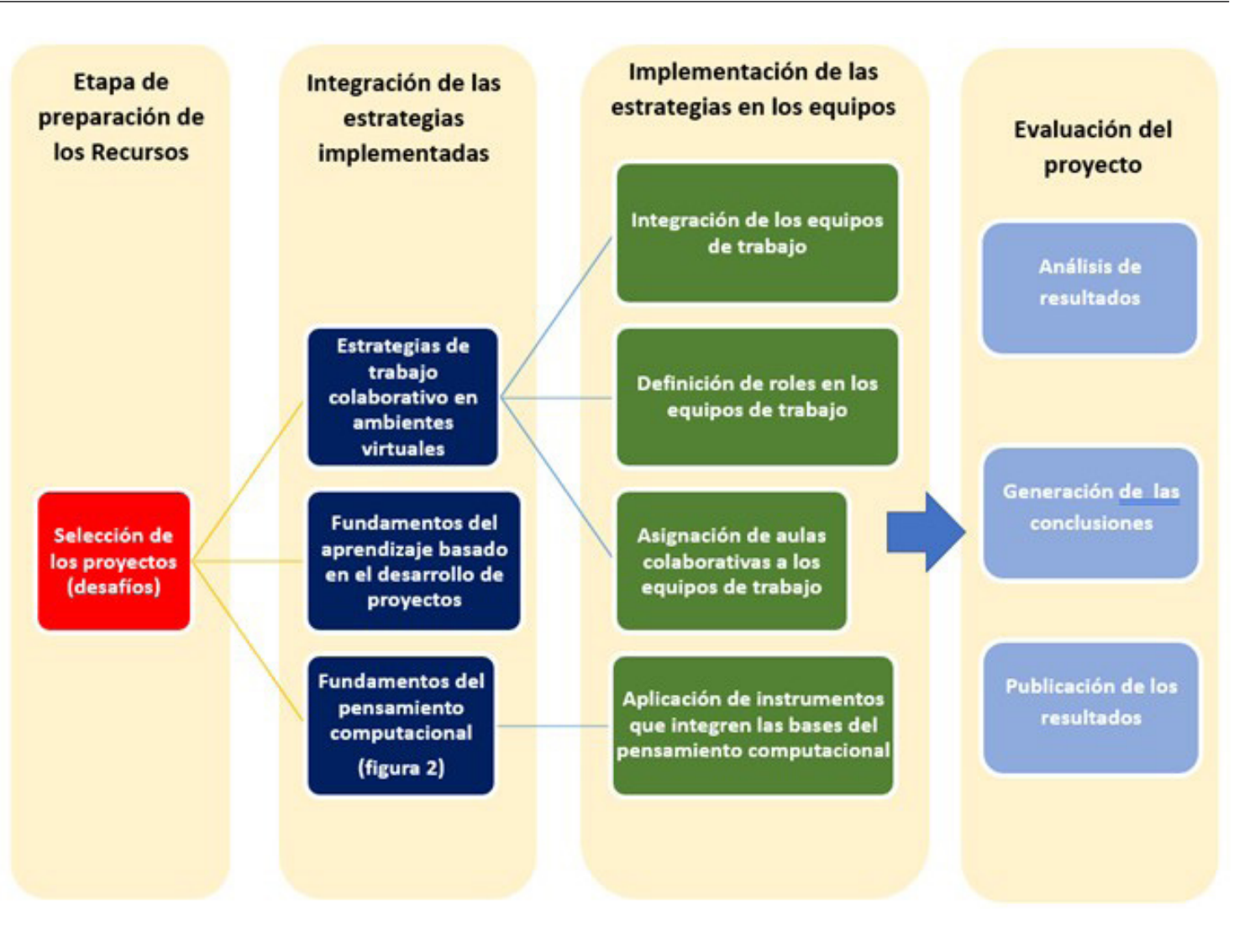

Figura 2. Competencias fundamentales asociadas al pensamiento computacional. Fuente: elaboración propia de los autores.
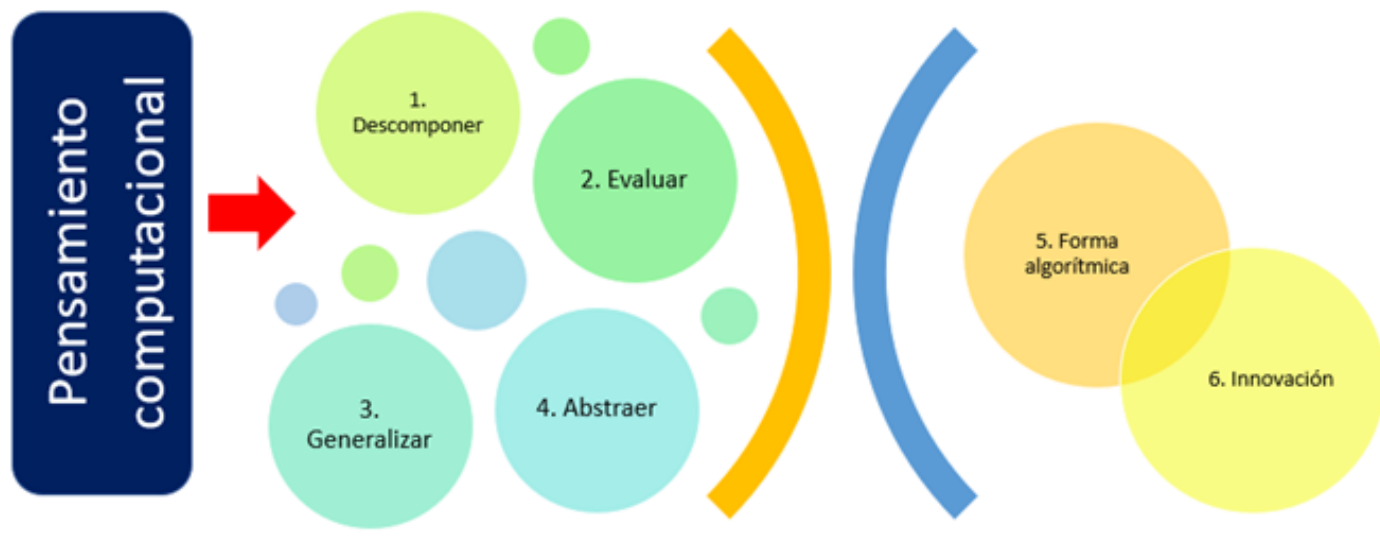

Fundamentos del pensamiento computacional 
Figura 3. Principios del trabajo colaborativo. Fuente: elaboración propia de los autores.

\section{Principios del trabajo colaborativo}

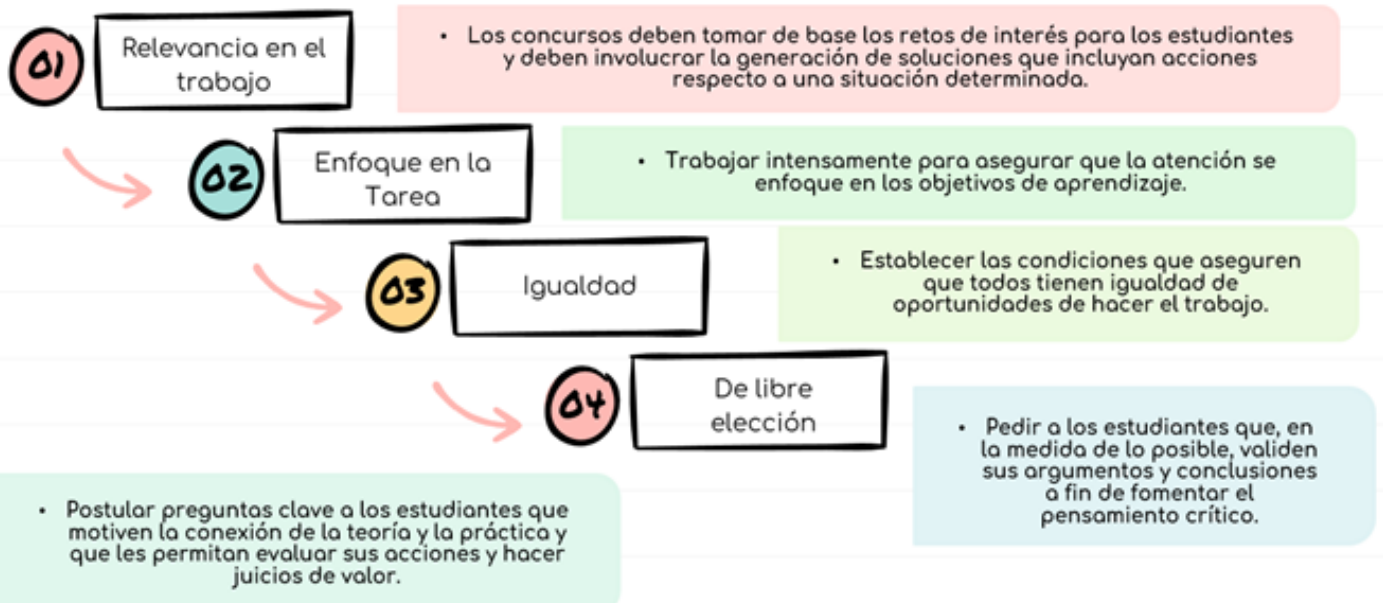

Figura 4. Implementación del trabajo colaborativo. Fuente: elaboración propia de los autores.

\section{Implementación de la estrategia}

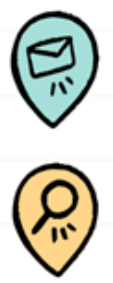

\section{Definición del proyecto}

Los alumnos deberán desarrollar un prototipo trabajando en equipo.

Integración de los equipos de trabajo

Los equipos fueron integrados con el docente a fin de garantizar que todos los equipos tuvieran iqualdad de condiciones para llevar a cabo la tarea.

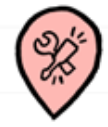

\section{Asignación de roles}

Una vez conformados los equipos, se deberá proceder a la asignación roles a cada miembro del equipo.

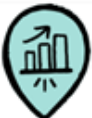

\section{Desarrollo del prototipo}

Los equipos deberán trabajar en el desarrollo del prototipo en aulas de espacio colaborativo.

Etapa final-presentación y evaluación del prototipo

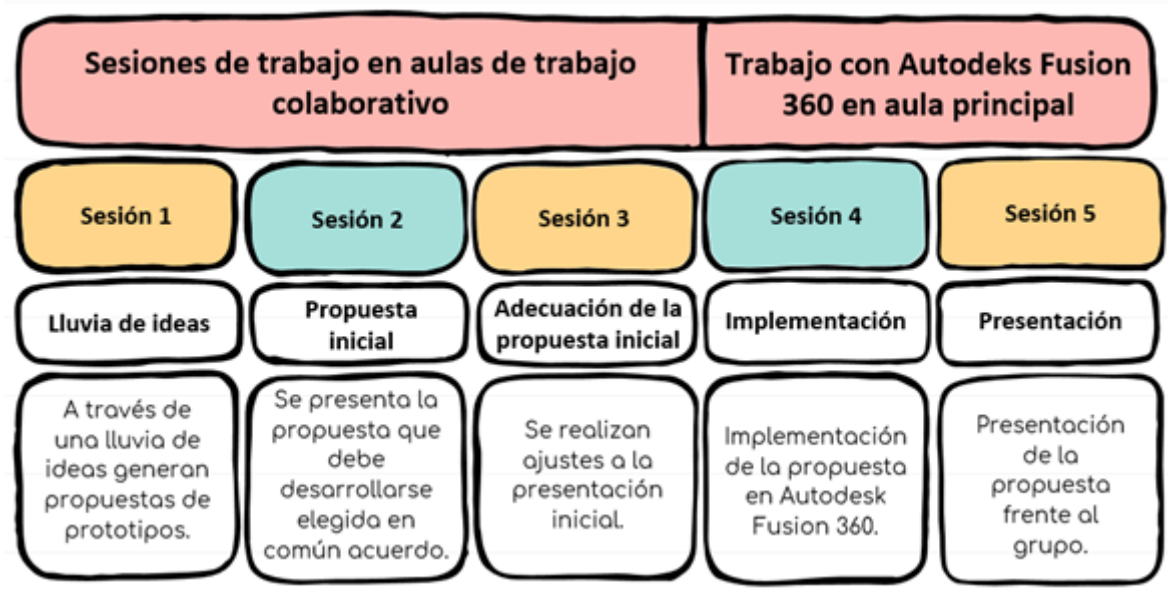


Figura 5. Asignación de roles en los equipos de trabajo. Fuente: elaboración propia de los autores.

\section{Roles al interior de los equipos}

Líder • Se asegurará de que todos puedan participar.

Líder . Dirigirá la discusión y enfocará el trabajo en la tarea.

Secretario : Escribirá los ideas y respuestos del grupo.

- En coso necesario, enviorá el documento escrito a cada miembro (usar el Chart Box).

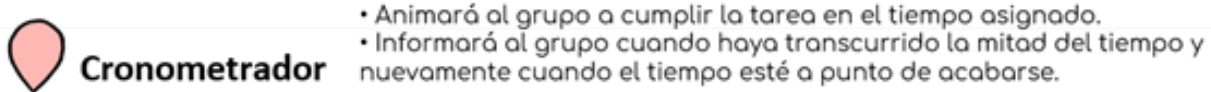

Cronometrador nuevamente cuando el tiempo esté o punto de acabarse.

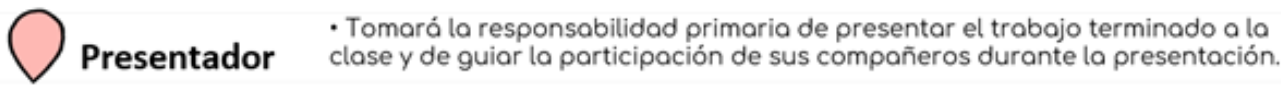

Figura 6. Indicadores de apropiación de los principios del pensamiento computacional. Fuente: elaboración propia de los autores.

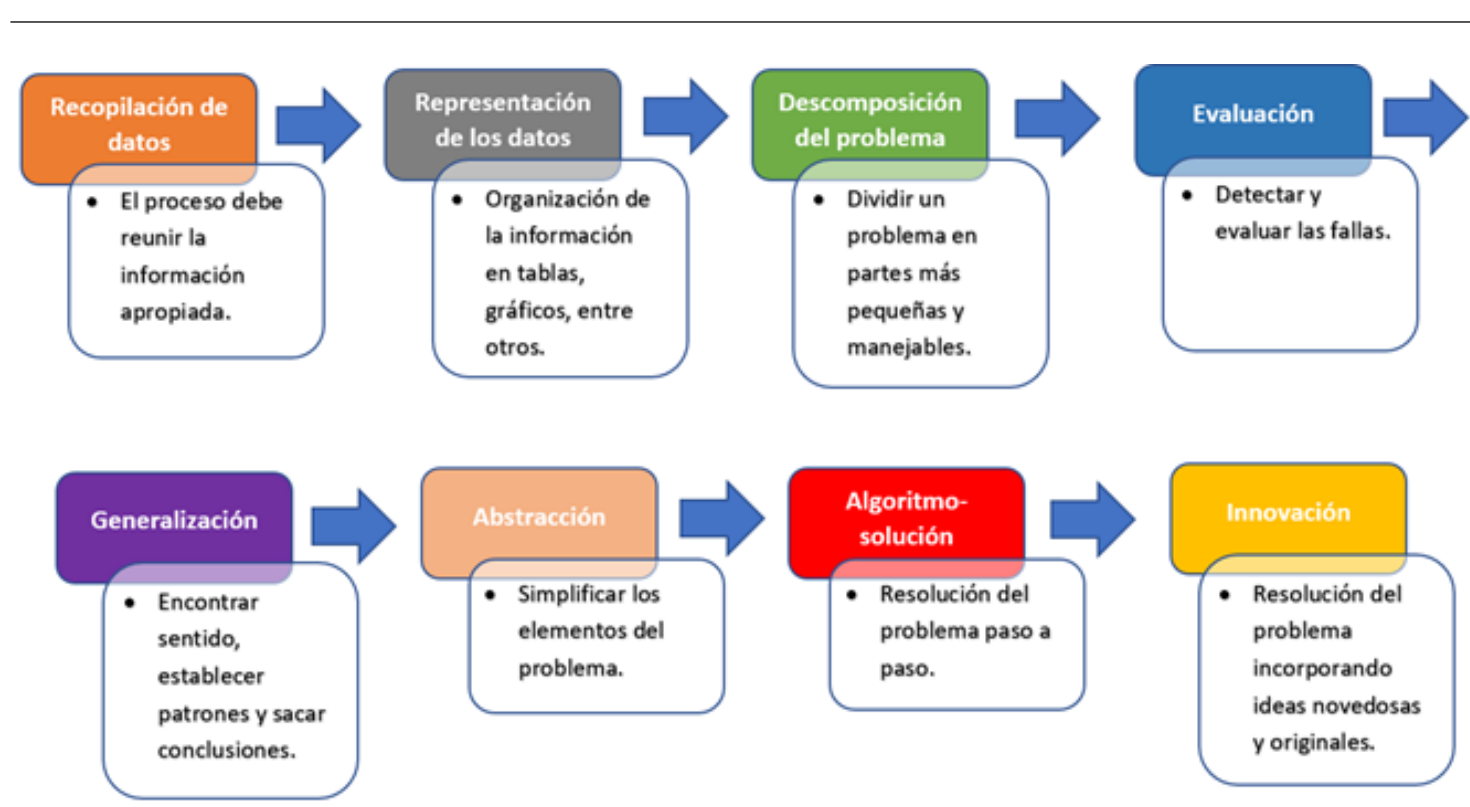

\section{Resultados}

\section{Proyecto 1}

Se planteó a los estudiantes el reto de identificar una necesidad presente en los alumnos de su nivel académico e institución educativa. Toda vez que identificaran una necesidad, debían proponer una solución a esta a través del diseño de un prototipo en Fu- 
sión 360, aplicando los principios del pensamiento computacional. A continuación, se presentan algunos de los diseños elaborados por los alumnos. Estos diseños incluyeron desde mesas sustentables para áreas verdes, sillas ergonómicas para el trabajo a distancia, bicicletas eléctricas para facilitar el traslado entre unidades académicas hasta juguetes antiestrés (figura 7).

Figura 7. Prototipos desarrollados por los alumnos en el primer proyecto. Fuente: elaboración propia de los autores.
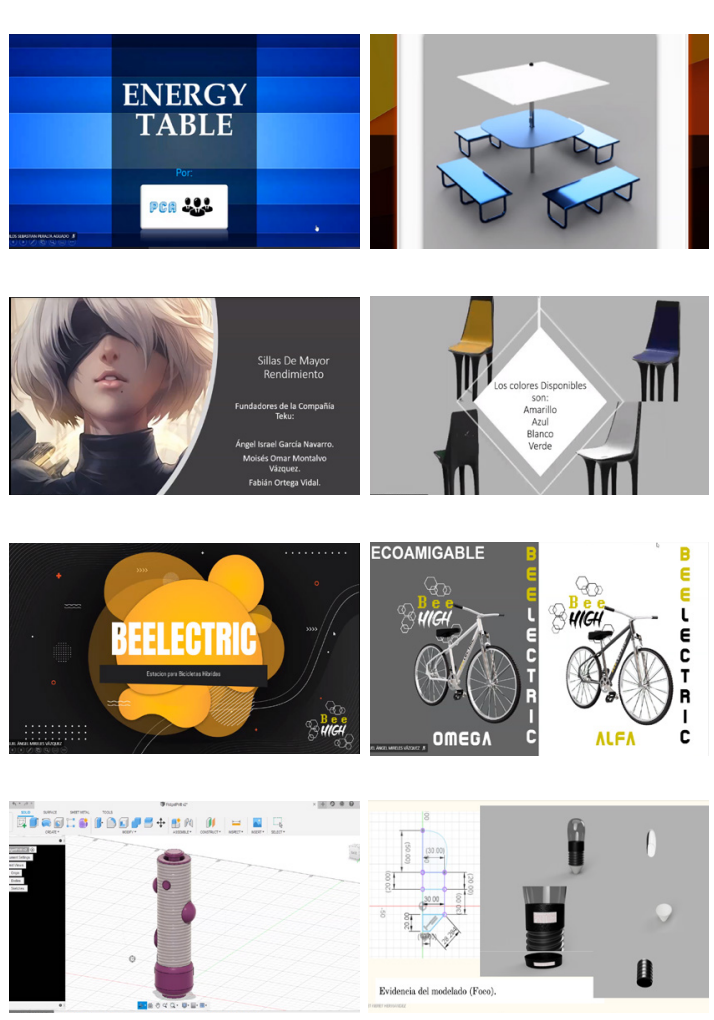

Durante el proceso de desarrollo de los prototipos, los alumnos tuvieron que reunir la información necesaria para cada diseño, organizar esta información en tablas y gráficos, dividir el problema en partes, hacer diseños iniciales para detectar y evaluar fallas, simplificar los elementos del problema y resolverlo incorporando ideas novedosas y originales. Cuando los diseños estuvieron terminados, los alumnos presentaron sus soluciones frente al grupo. Durante las presentaciones, los alumno se concentraron en dar respuesta a las siguientes preguntas ligadas al pensamiento crítico: ¿quién se beneficia del prototipo?, ¿cuáles son las fortalezas y debilidades del prototipo?, ¿dondé hay conceptos o situaciones similares a las que resuelve el prototipo?, ¿qué desafíos presenta la construcción del prototipo?, ¿por qué es este el mejor o el peor escenario? y ¿cómo se visualiza el prototipo en un futuro?

La evaluación de los prototipos se realizó empleando rúbricas basadas en los siguientes elementos: materiales de construcción, conocimiento del modelo, detalles del prototipo, recolección de datos, función del prototipo, adecuaciones (versiones) y el plano y modelo en Fusión 360. Durante las presentaciones, los alumnos, a su vez, contestaron cuestionarios digitales que les permitieron realizar una autoevaluación y una coevaluación del proyecto realizado.

\section{Proyecto 2}

Se pidió a los alumnos que diseñaran estructuras basadas en diferentes elementos geométricos y materiales a fin de analizar los esfuerzos presentes en dichas estructuras sometidas a cargas en modelos de simulación en 3D desarrollados en Fusión 360 (Education License). 
Del mismo modo que en el prrimer proyecto, los alumnos tuvieron que reunir la información necesaria para cada diseño, organizar esta información, dividir el problema en partes, hacer diseños iniciales para detectar y evaluar fallas, simplificar los elementos del problema y resolverlo incorporando ideas novedosas y originales.

Es importante destacar que el uso de simulaciones para el análisis de estructuras permite predecir las cargas y condiciones de servicio, calcular los esfuerzos correspondientes en los componentes estructurales y comparar estos esfuerzos con los esfuerzos críticos de falla a fin de garantizar la seguridad del diseño y el desempeño adecuado de la estructura, optimizando costos y tiempos destinados a la etapa de diseño (figuras 8 a 12) (Beer \& Johnston, 2010). Además, es preciso señalar que el uso de las simulaciones permite experimentar nuevas situaciones, que en la práctica resultarían costosas, o bien, que comprometerían la seguridad de las personas, por lo que no podrían ponerse a prueba. Este hecho contribuye al mejor entendimiento de los sistemas y al conocimiento y predicción acertada del comportamiento de estos.

Figura 8. Diseños estructurales con diferentes geometrías. Fuente: elaboración propia de los autores.
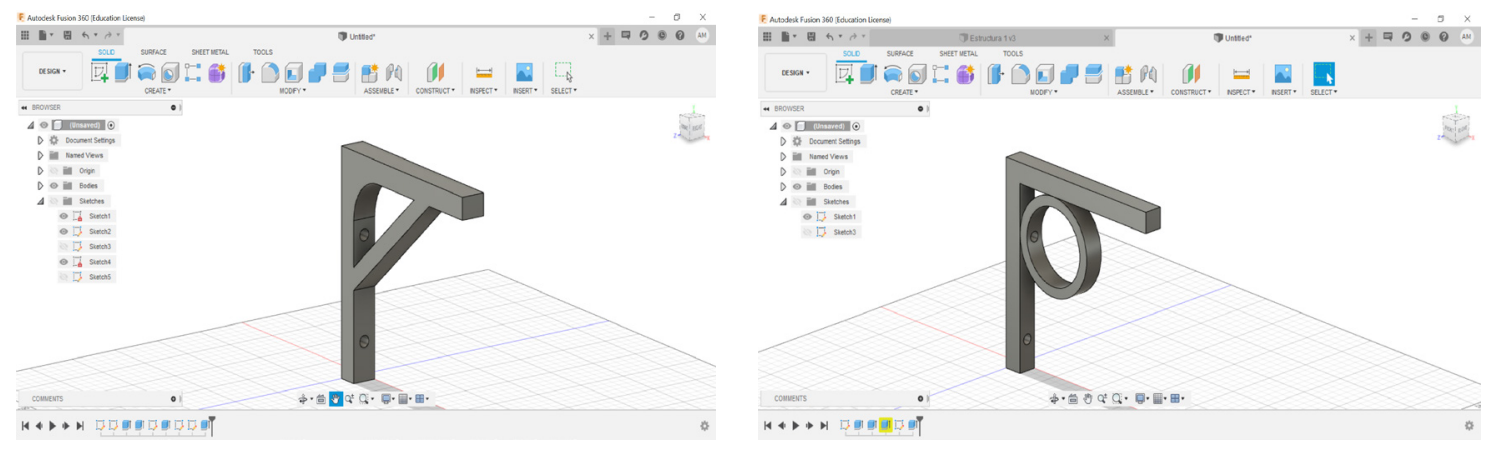

Figura 9. Asignación de cargas. Fuente: elaboración propia de los autores.

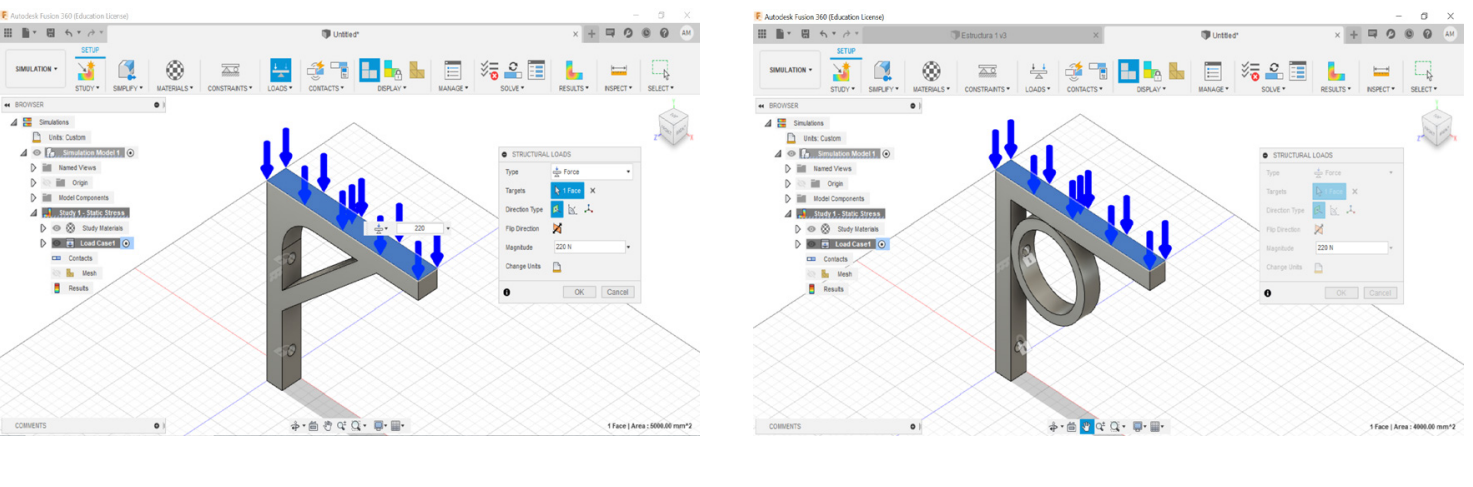


Figura 10. Revisión del equilibrio. Fuente: elaboración propia de los autores.

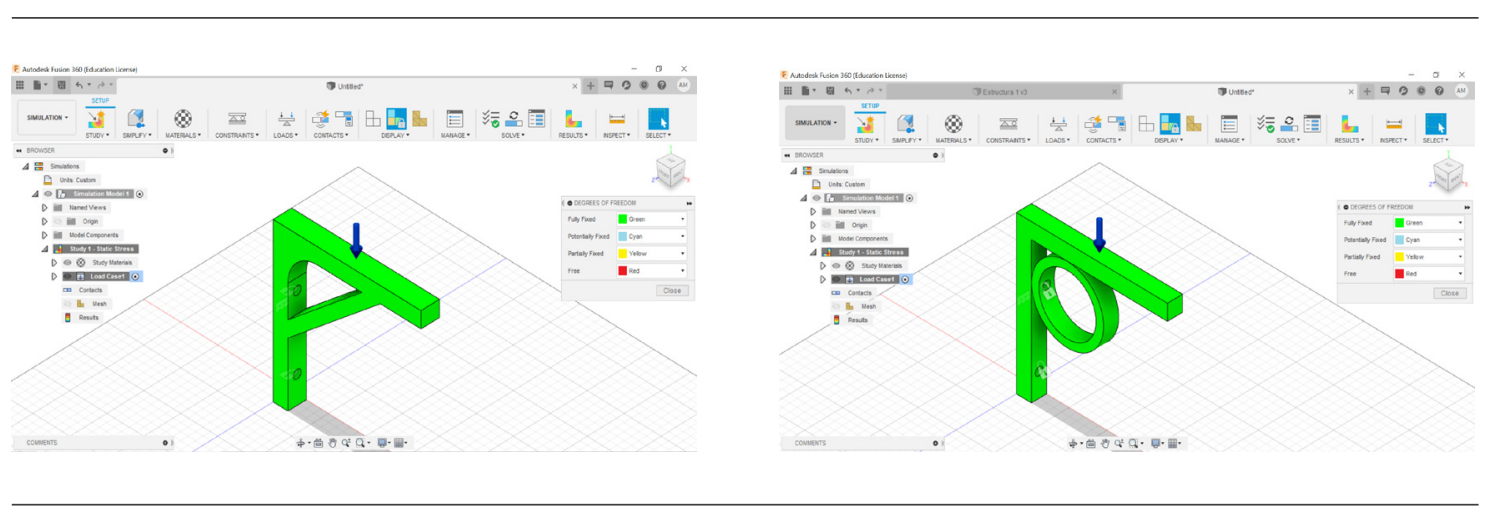

Figura 11. Evaluación del factor de seguridad. Fuente: elaboración propia de los autores.
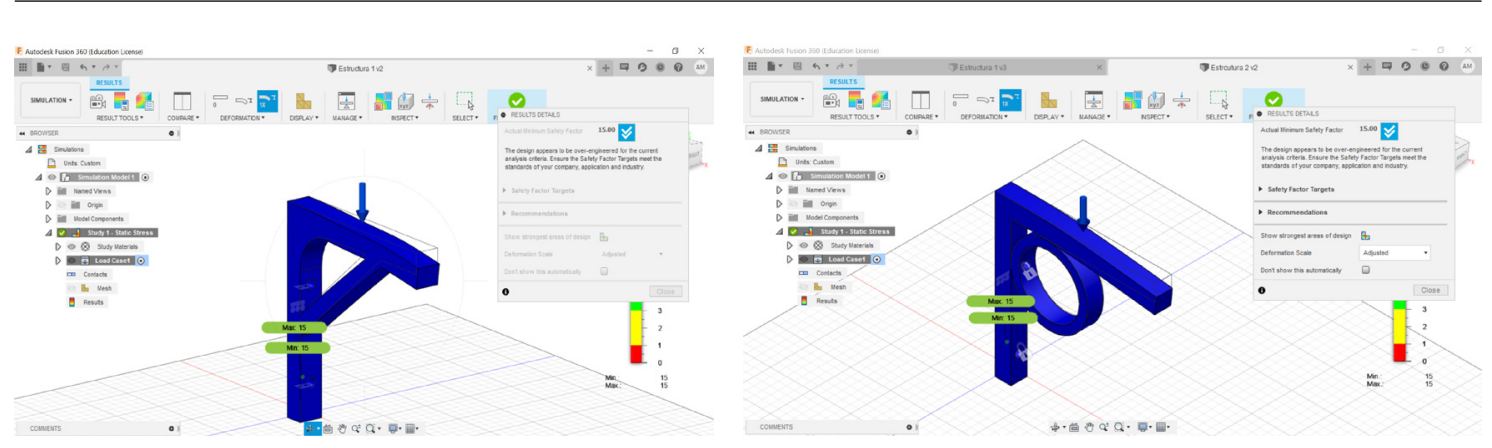

Figura 12. Evaluación de los esfuerzos y deformaciones. Fuente: elaboración propia de los autores.
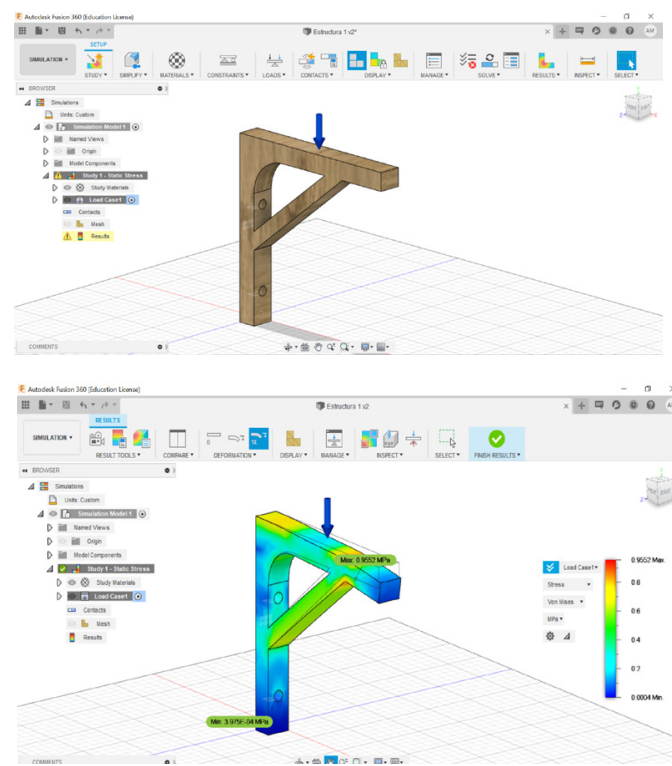
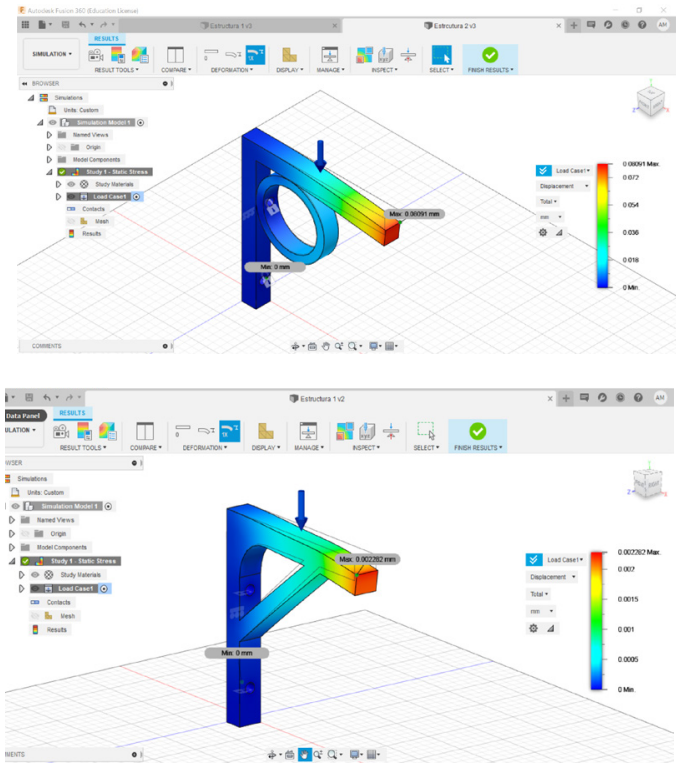
Cuando los diseños estuvieron terminados, nuevamente los alumnos presentaron sus soluciones frente al grupo. Durante las presentaciones, los alumno se concentraron en dar respuesta a las siguientes preguntas: ¿cuáles estructuras geométricas presentaron la mejor resistencia a la deformación?, ¿cuáles estructuras soportaron los mayores esfuerzos? ¿qué combinación de geometría y de material de construcción presentó el mejor desempeño? y ¿el diseño con el peor desempeño fue el que esparabas?

Al finalizar los proyectos, se les pidió a los alumnos que señalaran los indicadores de apropiación a los que, a su juicio, abonaron en mayor medida la realización de los proyectos. Ellos catalogaron los indicadores de mayor a menor contribución, asignando 1 al elemento que más impactó el desarrollo de proyectos y 6 al que tuvo menos impacto. Los resultados obtenidos se muestran en la figura 13.

\section{Discusión}

\section{Dimensión del alumno}

Actualmente, el pensamiento computacional está adquiriendo gran importancia debido a la evolución constante de la tecnología, lo que le ha permitido posicionarse como una actividad fundamental para el desarrollo de competencias relacionadas con el mundo laboral y personal de los estudiantes (Vázquez-Cano \& Ferrer, 2015). Al analizar la información recabada en los cuestionarios, se
Figura 13. Contribución del desarrollo de proyectos a la promoción de las competencias ligadas al pensamiento computacional.

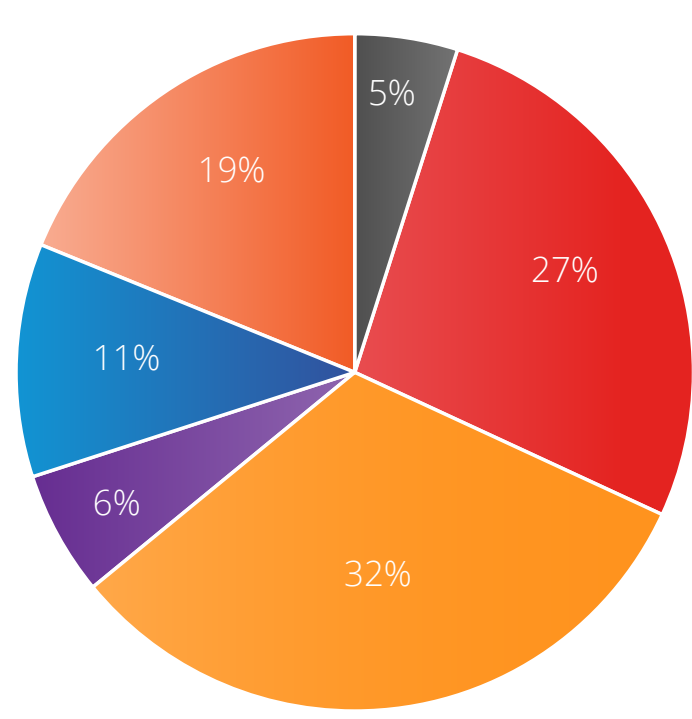

Recopilación y organización de la información

Descomposición y evaluación del problema

Generalización

Abstracción

Desarrollo del algoritmo solución

Innovación

observó que un 95\% de los alumnos consideró que estas actividades les permitieron desarrollar competencias ligadas a la búsqueda, selección y organización de la información, al trabajo coordinado y dirigido de las tareas asignadas, a la solución estructurada de los problemas y al análisis, evaluación, adecuación y presentación de los prototipos desarrollados. Como establecen Bocconi et al. (2016), estas competencias les serán de utilidad a los alumnos no solo desde el punto de vista laboral, sino también desde la óptica de una participación plena 
en la sociedad cada vez más digital hacia la que nos dirigimos.

La incorporación exitosa del pensamiento computacional en la educación requiere del impulso de la investigación en este campo a fin de que sea posible diseñar estrategias que apoyen a los docentes a integrar eficientemente los fundamentos del pensamiento computacional en los procesos educativos (Bocconi et al., 2016; Moreno-León, Román-González \& Robles, 2018) Es por ello por lo que los resultados obtenidos en el presente trabajo pueden servir de guía y motivación para la comunidad educativa a fin de que se pueda lograr la incorporación de los fundamentos del pensamiento computacional en el proceso de enseñanza-aprendizaje.

Así, los resultados de este trabajo muestran que el principal reto que identificaron los alumnos durante el desarrollo de los proyectos fue el de generar acuerdos al interior de los grupos que les permitieran hacer adecuaciones a los prototipos y llegar a sus versiones finales.

Es importante destacar que lo dicho anteriormente denota que los alumnos fueron expuestos a situaciones que emularon ambientes reales de trabajo en equipo, en donde ellos debían desarrollar competencias ligadas a la generación de consensos en ambientes diversos, competencias que les serán de utilidad para el resto de sus vidas. Esta necesidad de acuerdos también permitió a los alumnos reconocer la importancia de la comunicación efectiva en ambientes de trabajo colaborativo. Otro punto importante, que es preciso señalar, es que la mayor parte de los alumnos coincidió en que el uso de roles y la asignación de tareas a cada integrante les permitió realizar un verdadero trabajo colaborativo, en donde todos contribuyeron al cumplimiento de las tareas y la no presencialidad no fue un obstáculo para mantener un comunicación efectiva y una colaboración real en el desarrollo del proyecto.

Al finalizar los proyectos, a los alumnos se les pidió que señalaran los indicadores de apropiación a los que abonaron en mayor medida las actividades realizadas. En su mayoría coincidieron que las etapas de descomposición del problema, evaluación, generalización y abstraccción fueron las que les resultaron más díficiles de cubrir. Esto refuerza la idea de cuán importante es trabajar un enfoque de desarrollo de proyectos y solución de problemas que motive e impulse a los alumnos a transitar por las etapas de solución propuestas en el enfoque del pensamiento computacional (Vázquez-Cano \& Ferrer, 2015), evitando que los estudiantes quieran pasar mecánicamente de los datos al algoritmo de solución conocido.

Estudios previos desarrollados por el Ministerio de Educación y Formación Profesional en España han descubierto que el uso de simuladores computacionales en bachillerato ha contribuido al desarrollo del pensamiento computacional en los alumnos, incluso en mayor medida que los estudiantes que han trabajado los mismos contenidos haciendo uso de 
lenguajes de programación y dispositivos físicos de robótica habituales en este nivel educativo. Paralelamente, el uso de simuladores podría evitar que las instituciones educativas tengan que disponer de equipos físicos costosos, lo que abarataría y democratizaría la enseñanza de estas tecnologías (Moreno, 2018).

Si los docentes incluimos tareas que motiven al alumno a incorporar poco a poco las etapas de recopilación y organización de la información, de descomposición, evaluación, generalización y abstracción del problema y de diseño y aplicación de algoritmos novedosos y creativos, estaremos sin duda incidiendo positivamente en la motivación de los jovénes hacia temas STEM (Moreno, 2018) y, además, estaremos contribuyendo al desarrollo de competencias trascendentales en nuestros alumnos, que les serán útiles nos solo para su trayecto escolar, sino también a lo largo de su desarrollo personal y social.

\section{Dimensión del docente}

Es importante no perder de vista que, en el pensamiento computacional, el proceso que se sigue para la búsqueda de soluciones es lo verdaderamente trascendental; es decir, lo importante es que los alumnos aprendan a representar la realidad asociada a un problema y las expectativas y variables asocaidas a su solución, más que el aprendizaje de un lenguaje o un simulador (Álvarez, 2017). Una idea errónea que se puede tener del pensamiento computacional es creer que es un tema exclusivo de las personas asociadas al ámbito de la informática (Basogain et al., 2015). Como lo estableció Wing (2006), el potencial del pensamiento computacional radica en la forma de abordar los problemas, ya sea con la ayuda de ordenadores o bien con base en los procesos cognitivos de las propias personas. En este sentido, existe un claro interés y un esfuerzo creciente en incorporar el pensamiento computacional a los procesos educativos a través de proyectos, juegos, entornos de programación, entre otros (Basogain et al., 2015).

Así, a partir de la realización del presente trabajo, fue posible probar una estrategia de incorporación de los fundamentos del pensamiento computacional al proceso educativo a través del desarrollo de proyectos. Este trabajo permitió entonces reconocer la importancia que tiene el acercar los intereses de los alumnos al aula a fin de incrementar la motivación y lograr aprendizajes más significativos (Santos et al., 2015). Como docentes debemos promover que el alumno experimente un sentimiento de libertad durante la realización de las tareas a fin de incrementar su motivación. De igual modo, es importante destacar que, al interior del aula, los maestros debemos fomentar que los alumnos se integren a equipos de trabajo diferentes cada vez que se plantea un nuevo problema y que se definan roles al interior de los equipos para lograr el cumplimiento de la tarea e incrementar la interacción entre los alumnos. También es importante que al interior de nuestras aulas 
garanticemos que todos los equipos tengan igualdad de condiciones para realizar las actividades, partiendo del conocimiento del grupo, como lo establece Barkley (2010).

Durante el desarrollo de los proyectos, es importante fomentar el establecimiento de una conexión constante de la teoría y la práctica a fin de que los alumnos puedan evaluar sus acciones y hacer juicios de valor. En la conformación de los equipos podemos partir de un esquema inicial, en el cual el docente construye los grupos de trabajo cuidando que todos tengan igualdad de condiciones de cumplir con la tarea o proyecto, y conforme se van asignando nuevas actividades, se puede promover que los alumnos cambien de equipo, incrementando con ello la interacción alumno-alumno. Por útlimo, es importante considerar que ciertos procesos en ambientes virtuales son más lentos que en la presencialidad, por lo que los docentes debemos servir de guía y de soporte a los alumnos sin que esto implique acelerar los procesos de enseñanza-aprendizaje.

Finalmente, es importante mencionar que la integración de los fundamentos del pensamiento computacional en los procesos educativos requiere romper con la metodología tradicional. Además, demanda experiencia, formación y capacitación continua de la planta docente (González, 2019). Una perspectiva de mejora de la estrategia seguida en el presente trabajo sería la creación de proyectos multidisciplinarios que involucren diferentes asignaturas, en las que se ejerciten diversas competencias. Es importante que las experiencias de los docentes se registren desde el enfoque relacional, epistemológico y pragmático a fin de fortalecer esta estrategia.

\section{Conclusiones}

El planteamiento de retos, problemas o desafíos que impliquen la generación de soluciones a partir del desarrollo de proyectos en equipos de trabajo de diversos genera un espacio idóneo para que los alumnos asuman roles, se comuniquen eficientemente, aprendan entre ellos y desarrollen soluciones innovadoras bajo un ambiente real de aprendizaje colaborativo. A partir del presente proyecto, fue posible observar que el trabajo en pequeños grupos potencia el aprendizaje al permitir un mayor nivel de interacción estudiante-estudiante, estudiante-profesor y estudiante-contenido. Al trabajar en grupos colaborativos, los estudiantes aprenden de sus pares y se ayudan y motivan unos a otros a fin de de lograr las tareas encomendadas. Sin embargo, es importante señalar que los docentes debemos dedicar tiempo suficiente a pensar y plantear retos que sean de interés para los alumnos. En la medida en que los retos sean más interesantes, lograremos aprendizajes más significativos, mejores niveles de retención del conocimiento y mayores niveles de interacción entre los estudiantes.

También es necesario que, como docentes, nos enfoquemos en promover ambientes que les permitan a nues- 
tros alumnos desarrollar competencias que les sean de utilidad para su vida personal y profesional. Algunas de estas competencias tienen relación directa con la forma en la que los estudiantes abordan los problemas. En este sentido, debemos promover que los alumnos, cuando se enfrenten a un problema, lejos de frustrarse, encuentren soluciones basadas en la búsqueda y organización de información, en el análisis y evaluación de problemas y en la generalización y diseño de soluciones innovadoras y creativas.

Para lograr esto, debemos promover en el aula ambientes que favorezcan el desarrollo de estas competencias; de tal forma que, paso a paso, los estudiantes vayan reconociendo sus propios procesos creativos e integrando habilidades que les permitan trabajar en grupos diversos y generar canales de comunicación que hagan posible el cumplimiento de las tareas. La incorporación de los principios del pensamiento computacional al desarrollo de proyectos y a la solución de problemas, sin duda alguna, abona al logro de este objetivo.

Por útlimo, el desarrollo de proyectos con base en el trabajo colaborativo y en los principios del pensamiento computacional permitirá el desarrollo de competencias socioemocionales ligadas a la confianza mutua, la comunicación eficaz, la gestión adecuada de conflictos, el reconocimiento y valoración de las diferencias, el valor de la toma de decisiones y el reconocimiento de los objetivos del grupo sobre los individuales.

\section{Información complementaria}

Agradecimientos a las direcciones de la Escuela de Nivel Medio Superior de León de la Universidad de Guanajuato, del Instituto Tecnológico y de Estudios Superiores de Monterrey, Campus León, y de la Unidad Profesional Interdisciplinaria de Ingeniería, Campus Guanajuato, Instituto Politécnico Nacional, por el apoyo brindado para la realización del presente proyecto.

\section{Referencias}

Álvarez, M., (2017). Desarrollo del pensamiento computacional en educación primaria: una experiencia educativa con Scratch. UTE-Revista de Ciències de l'Educació, 2, 45-64. https://doi. org/10.17345/ute.2017.2.1820

19

Barkley, E. \& Howell, C. (2010). Student Engagement Techniques: A Handbook for College Faculty. Wiley.

Basogain, X., Olabe M. A. \& Olabe, J. C. (2015). Pensamiento Computacional a través de la Programación: Paradigma de Aprendizaje. RED-Revista de Educación a distancia, 46(6), 1-33. https:// doi.org/10.6018/red/46/6

Beer, F., Johnston, E. R., Mazurek, D. \& Eisenberg, E. (2010). Mecánica vectorial para ingenieros. Estática. 9. a ed. Mc Graw Hill.

Bocconi, S., Chioccariello, A., Dettori, G., Ferrari, A. \& Engelhardt, K. (2016). Developing computational thinking in compulsory education-Implications for policy and practice. Joint Research Centre. https://doi.org/10.2791/792158

Caballero, Y.A. \& García-Valcárcel, A. (2017). Development of computa- 
Promación de las competencias ligadas al pensamienta computacional a través del aprendizaje basado en el desarrollo de prayectos

tional thinking skills and collaborative learning in initial education students through educational activities supported by ICT resources and programmable educational robots. En: Proceedings of the 5th International Conference on Technological Ecosystems for Enhancing Multiculturality (pp. 1-6). ACM. doi.org/10.1145/3144826.3145450

Durak H. \& Saritepeci M. (2018). Analysis of the relation between computational thinking skills and various variables with the structural equation model. Computers \& Education, 116, 191202, https://doi.org/10.1016/j.compedu.2017.09.004

Durán Velásquez, L. (2018). Estrategia de gestión basada en el cuadro de mando integral para la empresa Herrametal C.A. ubicada en municipio Iribarren, Estado Lara. Enfoque Disciplinario, 3(1), 49-71. Recuperado a partir de http://enfoquedisciplinario.org/revista/index.php/enfoque/article/view/12

García, D. (2012). The Beauty and Joy of Computing. University of California. https://bjc.berkeley.edu/

García-Utrera L, Figueroa-Rodríguez S. \& Esquivel I. (2014). Modelo de Sustitución, Aumento, Modificación y Redefinición (SAMR): Fundamentos y aplicaciones. En: Los Modelos Tecno-Educativos: Revolucionando el aprendizaje del sig/o XXI (pp. 205-220). DSAE-Universidad Veracruzana.

González, E. (2019). Desarrollo de Competencias STEM y pensamiento computacional con Scratch a través de metodologías activas [Tesis de maestría Universidad de Nebrija]. Repositorio Facultad de Letras y Educación, Universidad de Nebrija. https://doi. org/10.13140/RG.2.2.36410.75208

Liu, H. P., Perera, S. M. \& Klein, J. W. (2017). Using Model-Based Learning to Pro- mote Computational Thinking Education. En: Emerging Research, Practice, and Policy on Computational Thinking (pp. 153-172). Springer. https://doi. org/10.1007/978-3-319-52691-1_10

Morales Espíndola, M. G., Moreno Cortés, K. C., Romano Cadena, M. M. del S., \& García Alarcón, M. del R. (2020). Gestión del conocimiento, a través de plataformas y herramientas digitales de aprendizaje ante la migración de clases presenciales a en linea. Revista GEON (Gestión, Organizaciones Y Negocios), 7(2), 1-19. https://doi. org/10.22579/23463910.217

Moreno-León, J., Román-González, M. \& Robles, G. (2018). Comparing Computational Thinking Development Assessment Scores with Software Complexity Metrics. En: IEEE Global Engineering Education (pp. 1040-1045).EDUCON. 10.1109/EDUCON.2016.7474681

Rivera Cerpa, Y. M., \& Conrado Tobón, J. (2016). Impacto de los líderes en la productividad de las empresas de servicio de aseo en la ciudad de Barranquilla. Dictamen Libre, (19), 57-68. https://doi.org/10.18041/2619-4244/ dl.19.2901

Santos, A. R., Sales, A., Fernandes, P. \% Nichols, M. (2015). Combining Challenge-Based Learning and Scrum Framework for Mobile Application Development. En: Proceedings of the 2015 ACM Conference on Innovation and Technology in Computer Science Education (pp. 189-194). ACM. https://doi. org/10.1145/2729094.2742602

Vázquez- Cano, E. \& Ferrer, F. (2015). La creación de videojuegos con Scratch en educación secundaria. Communication Papers: Media Literacy and Gender Studies, 4(6), 63-73. 
Wing, J. M. (2006). Computational Thinking. Communications of the ACM, 49(3), 33-35. https://doi. org/10.1145/1118178.1118215

Zapotecatl, J. (2018). Introducción al pensamiento computacional: Conceptos básicos para todos. Amexcop, Academia Mexicana de Computación. 